Review Article

\title{
Focus on Mesenchymal Stem Cell-Derived Exosomes: Opportunities and Challenges in Cell-Free Therapy
}

\author{
Lin Cheng, ${ }^{1}$ Kun Zhang, ${ }^{2}$ Shuying Wu, ${ }^{1}$ Manhua Cui, ${ }^{1}$ and Tianmin $\mathrm{Xu}^{1}$ \\ ${ }^{1}$ Gynecology and Oncology Department of the Second Hospital of Jilin University, Ziqiang Street 218, Changchun 130000, China \\ ${ }^{2}$ Medical Research Center, Second Clinical College, Jilin University, Ziqiang Street 218, Changchun 130000, China \\ Correspondence should be addressed to Tianmin Xu; xutianmin@126.com
}

Received 11 August 2017; Revised 5 November 2017; Accepted 22 November 2017; Published 19 December 2017

Academic Editor: Changwon Park

Copyright (c) 2017 Lin Cheng et al. This is an open access article distributed under the Creative Commons Attribution License, which permits unrestricted use, distribution, and reproduction in any medium, provided the original work is properly cited.

\begin{abstract}
Mesenchymal stem cells have been at the forefront of regenerative medicine for many years. Exosomes, which are nanovesicles involved in intercellular communication and the transportation of genetic material transportation that can be released by mesenchymal stem cells, have been recently reported to play a role in cell-free therapy of many diseases, including myocardial infarction, drug addiction, and status epilepticus. They are also thought to help ameliorate inflammation-induced preterm brain injury, liver injury, and various types of cancer. This review highlights recent advances in the exploration of mesenchymal stem cell-derived exosomes in therapeutic applications. The natural contents, drug delivery potency, modification methods, and drug loading methods of exosomes are also discussed.
\end{abstract}

\section{Introduction}

Mesenchymal stem cells (MSCs) originate from the mesoderm of many tissues, including bone marrow, liver, spleen, peripheral blood, adipose, placenta, and umbilical cord blood, and have the capacity to self-renew and the ability to generate differentiated cells. Over the last decade, MSCs have emerged as a popular research topic because of their potential role in regenerative medicine, immunoregulation, neuroprotection, and antitumor effects originally attributed to direct cell replacement. However, experimental data indicates that most MSCs are largely cleared, while a small proportion will integrate into injured tissue after intravenous injection [1]. Furthermore, the "cell replacement theory" does not account for the sufficient durations in a variety of disease models [2, 3]. Recently, several mechanisms have been put forward regarding the therapeutic potential of MSCs, including (1) paracrine factors involving proteins/peptides and hormones and (2) the transfer of mitochondria or exosomes/microvesicles packaging multitudinous molecules [4].

Exosomes are a family of nanoparticles with a diameter in the range of $40-100 \mathrm{~nm}$ that are generated inside multivesicular endosomes or multivesicular bodies (MVBs) and are secreted when these compartments fuse with plasma membrane [5]. Exosomes are enriched in endosomederived components as well as many bioactive molecules such as proteins, lipids, mRNAs, microRNAs (miRNAs), long noncoding RNAs (lncRNAs), transfer RNA (tRNA), genomic DNA, cDNA, and mitochondrial DNA (mtDNA) [6-12]. It has also been reported that exosomes may be released from multiple cell types, including reticulocytes [13], immunocytes, tumor cells, and MSCs [14]. This suggests that the secretion of exosomes is a general cellular function that plays an important role in the intercellular transfer of information.

In this review, we focus on the mechanisms of exosomes/ microvesicles, covering the current knowledge on biological characteristics and their potential cell-free therapeutic applications for MSC-derived exosomes.

\section{Characterization and Isolation of Exosomes}

Exosomes were first discovered by Harding's group as "a garbage can" in maturing sheep reticulocytes [13]. Originally, they were thought to have a typical "cup-shaped" or "saucerlike" morphology when analyzed by electron morphology 
TABLE 1: Characterization of various extracellular vesicles.

\begin{tabular}{lccc}
\hline & Exosomes & MVs & Apoptotic bodies \\
\hline Size & $30-100 \mathrm{~nm}$ & $50-2000 \mathrm{~nm}$ & $500-4000 \mathrm{~nm}$ \\
Surface markers & CD63 & ARF6 & TSP \\
(used most) & CD9 & VCAMP3 & C3b \\
\hline
\end{tabular}

$[15,16]$. Zabeo's group revealed a wide diversity in exosome morphology when purified from homogeneous cell types (the human mast cell line HMC-1). They classified exosome morphology into nine categories: (1) single vesicle; (2) double vesicle; (3) triple vesicle or more; (4) small double vesicle; (5) oval vesicle; (6) small tubule; (7) large tubule; (8) incomplete vesicle; and (9) pleomorphic vesicle [17]. This categorization suggested that different morphologies of exosomes may be accompanied by various and specific functions. Exosomes also contain surface proteins unique to the endosomal pathway, which are generally used to characterize exosomes and distinguish them from microvesicles (MVs), apoptotic bodies, and other vesicles (Table 1), such as tetraspanins (CD63, CD81, and CD9), heat shock proteins (Hsc70), lysosomal proteins (Lamp2b), the tumor-sensitive gene 101 (Tsg101), and fusion proteins (CD9, flotillin, and annexin) $[12,18]$. Exosomes are released in almost all types of extracellular fluids, including blood, urine, amniotic fluid, ascites, hydrothorax, saliva, breast milk, seminal fluid, and cerebrospinal fluid. Exosomal content greatly depends on cellular origin. For example, exosomes derived from B lymphocytes that bring functional MHCI, MHCII, and $\mathrm{T}$ cell costimulatory molecules can stimulate $\mathrm{T}$ cell proliferation [19]. Furthermore, cancer cell-derived exosomes contain gelatinolytic enzymes and other cell adhesionrelated molecules to help tumor progression and metastasis [20]. Importantly, these cancer cell-derived exosomes are actively incorporated by MSCs in vitro and in vivo, in that the transfer of exosomal proteins and miRNAs acquire the physical and functional characteristics of tumor-supporting fibroblasts [21, 22]. For more details on the molecular cargos and extracellular signal transmission pathway of exosomes, the reader may refer to ExoCarta (http://www.exocarta.org) or EVpedia (http://evpedia.info), as well as the American Society for Exosomes and Microvesicles (http://www.asemv. org), for an in-depth exploration.

Ultracentrifugation and a commercial kit rooted in polymer-based precipitation are the most well-established purification protocols [16]. Other conventional validated isolation methods described in the literature include ultrafiltration, chromatography, and affinity capture [23]. New protocols have been established in order to facilitate the large-scale and high-purity manufacture of exosomes. Microfluidic techniques [24] are based on electrochemical, electromechanical, viscoelastic [25], optical, nonoptical, and other principles, yet the isolation is a mixed population of small nanoparticles without further demonstration of their intracellular origin. Thus, we use the term exosomes in this review to refer to extracellular vesicles characterized by exosome-specific surface markers, regardless of the primitive appellations in the published data.

\section{Cargos and Functions of MSC-Derived Exosomes}

The abundance of cargos identified from MSC-derived exosomes attracts broad attention because of their therapeutic potential in cardiovascular disease, tissue (kidney, liver, skin, and cornea) repair, immune disease, tumor inhibition, and neurological disease (Figure 1). They function largely via the constant transfer of miRNAs and proteins, resulting in the alteration of a variety of activities in target cells via different pathways.

3.1. Protein. Over 900 species of proteins have been collected from MSC-derived exosomes according to ExoCarta. With the exception of some common proteins involved in cell metabolism and the cytoskeleton, many proteins have been found in different tissue sources of MSC-derived exosomes. Proteomic studies by Kang's group identified 103 proteins from neural stem cell-derived exosomes. For example, the presence of polymyositis/scleroderma autoantigen 2 (PM/Scl2), a highly specific nuclear autoantigen, indicates that exosomes may be involved in triggering autoimmunity. They also found an imparity between exosomes larger than the baseline $(50 \mathrm{~nm})$ and those of smaller morphology [26]. These findings may explain the phenomenon recently observed by Caponnetto et al. regarding size-dependent cellular uptake of exosomes by target cells [27]. Intriguingly, all enzymes involved in the ATP synthesis of glycolysis (glyceraldehyde 3-phosphate dehydrogenase (GAPDH), phosphoglycerate kinase (PGK), phosphoglucomutase (PGM), enolase (ENO), and pyruvate kinase $\mathrm{m} 2$ isoform (PKm2)), as well as the rate-limiting glycolytic enzyme phosphorylated PFKFB3 that upregulates phosphofructose kinase, were identified in MSC-derived exosomes. Furthermore, oxidative stress was reduced via peroxiredoxins and glutathione Stransferases in MSC-derived exosomes [28], which suggests that replenishing glycolytic enzymes to increase ATP production, as well as additional proteins to reduce oxidative stress through exosomal transportation, may help reduce cell death in myocardial ischemia/reperfusion injury. Comparable levels of VEGF, extracellular matrix metalloproteinase inducer (EMMPRIN), and MMP-9 have also been reported in MSC-derived exosomes. These three proteins play a vital role in stimulating angiogenesis [29], which could be fundamental for tissue repair. Recent experimental evidence summarized by Burrello suggests that transcriptional factors, such as Nanog, octamer-binding transcription factor 4 (Oct-4), HoxB4, and Rex-1, play an important role in the immune system [30]. For example, HoxB4 has been shown to affect DC maturation and T-cell proliferation, differentiation, and activation through WNT signaling. Interestingly, membrane proteins and exosome-specific surface markers, such as CD81, CD63, and CD9, may affect the immune response by regulating cell adhesion, motility, activation, and signal transduction [31]. Several studies have also shown that exosomes derived from MSCs harbor cytokines and growth factors, such as TGF $\beta 1$, interleukin-6 (IL-6), IL-10, and hepatocyte growth factor (HGF), which have been proven to contribute to immunoregulation [30]. 


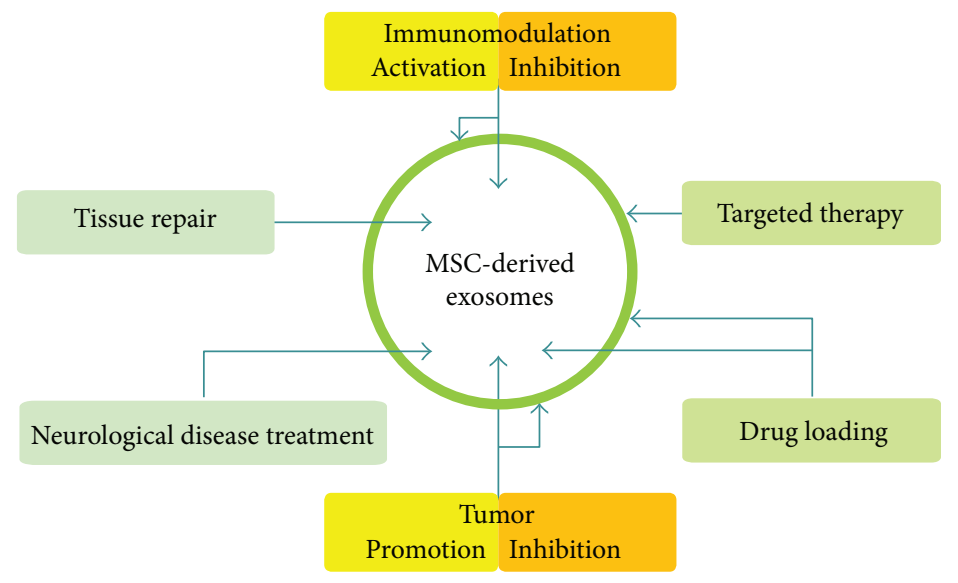

FIgURE 1: The main functions of MSC-derived exosomes. The external bilayer (green circle) is the membrane and the internal bilayer (white circle) is packed with various bioactive compounds.

3.2. miRNAs. miRNAs consist of a class of small noncoding RNAs that regulate gene expression posttranscriptionally by targeting mRNAs to induce suppression of protein expression or cleavage [32]. Many miRNAs have been found in MSC-derived exosomes and are reportedly involved in both physiological and pathological processes such as organism development, epigenetic regulation, immunoregulation, tumorigenesis, and tumor progression. Notably, exosomes with membrane structure act as preservers and deliverers of miRNAs, transferring functional miRNAs into recipient cells. It has been reported that exosomal miR-23b, miR451, miR-223, miR-24, miR-125b, miR-31, miR-214, and miR-122 [33, 34] may inhibit tumor growth and stimulate apoptosis through different pathways. For instance, miR$23 \mathrm{~b}$ promotes dormancy in metastatic breast cancer cells via the suppression of the target gene MARCKS, which encodes a protein that promotes cell cycling and motility [34]. MiR-16, shuttled by MSC-derived exosomes, has also been found to suppress angiogenesis by downregulating VEGF expression in breast cancer cells [35]. Recently, let7f, miR-145, miR-199a, and miR-221, which are released from umbilical MSC-derived exosomes, have been found to largely contribute to the suppression of hepatitis $\mathrm{C}$ virus (HCV) RNA replication [36]. Di Trapani's group evaluated the immunomodulatory effects exerted by MSC-derived exosomes on unfractionated peripheral blood mononuclear cells and purified T, B, and NK cells. They observed that exosomes had higher levels of miRNAs compared to MSCs and could also induce inflammatory priming via increasing levels of miR-155 and miR-146, which are two miRNAs involved in the activation and inhibition of inflammatory reactions [37]. Similar immunosuppressive functions have also been reported in animal experiments by Cui et al. [38]. Exosomes from MSCs effectively increased the level of miR-21 in the brain of AD mice. Additionally, replenishment of miR-21 restored the cognitive deficits in APP/PS1 mice and prevented pathologic features by regulating inflammatory responses and restoring synaptic dysfunction [38]. Recent studies have also shown that aging is substantially controlled by hypothalamic stem cells, partially through the release of exosomal miRNAs [39]. However, contradictions regarding these outcomes remain. A quantitative analysis of exosomal miRNA abundance and stoichiometry by Chevillet's group quantified both the number of exosomes and the number of miRNA molecules in replicate samples isolated from diverse sources. Regardless of the source, the study indicated that, on average, over 100 exosomes would need to be examined to observe one copy of a given abundant miRNA, suggesting that most individual exosomes do not carry biologically significant numbers of miRNAs and are thus unlikely to be functional individually as vehicles for miRNA-based communication [40].

3.3. Others. In 2006, MSC-derived exosomes that could modulate the phenotype of target cells, supporting self-renewal of hematopoietic progenitors and multipotency by transfer of growth factors and mRNA, were first reported. For instance, exosomal SOX2 was found to initiate innate responses against microbial infection through neutrophil activation [41]. Although MSC-derived exosomes have the same morphology as exosomes from other cells and carry typical markers, they are quite different in regard to compartmentalization and protein and RNA composition. For example, studies have indicated that not all MSC-derived exosomes are equivalent [42]. Baglio's group [6] characterized the small RNAome of exosomes released by early passage adipose-MSCs (ASC) and bone marrow-MSCs (BMSCs). They found a large discrepancy in the proportion of miRNAs in total small RNA content between cells (19-49\%) and exosomes $(2-5 \%)$, suggesting that the miRNAs in exosomes do not merely reflect the cellular content. Further studies regarding the overrepresentation of small RNA contenttRNAs revealed a similar outcome. The most abundant tRNA in ASC exosomes, tRNA GCC (Gly), represented only a small fraction $(5 \%)$ of the total cellular tRNA. Importantly, the authors also determined that the striking differences in tRNA species seemed to be associated with the differentiation status of MSCs. Recent research has shown that the stability of exosome composition is susceptible to localized environmental conditions. For example, hypoxia and inflammatory signals, such as lipopolysaccharides, may be strong interference factors [43]. 


\section{Exosomes as Drug Delivery Vehicles}

Optimal features of drug delivery vehicles may be applied to improve carrier qualities, including cellular tropism, efficient therapeutic cargos, appropriate physicochemical properties, and sufficient immune tolerance. Among the many drug platforms, liposomes have been the preferred pharmaceutical vehicles for drug delivery. A wide range of liposome products have been approved for the treatment of diseases, including fungal infections, pain management, hepatitis A, influenza, and various types of cancer [44, 45]. In contrast to liposomes, exosomes are optimal for drug delivery because of their natural properties and plasticity with minor modifications. Here, we compare exosomes and liposomes and suggest that exosomes may be a promising star for drug delivery.

4.1. Exosomes versus Liposomes. Exosomes and liposomes are both coated with a phospholipid membrane. The membrane structure of exosomes is inlayed with multiple natural biomolecules, such as surface proteins and MHCs, while liposomes may be modified with targeting ligands or inert polymeric molecules such as oligosaccharides, glycoproteins, polysaccharides, and synthetic polymers [45]. The size of liposomes is in the range of $30 \mathrm{~nm}$ to several microns [46]. Smaller liposomes (as small as exosomes) display a prolonged circulation time compared to larger ones, but the capacity for optimal drug reservation and release profiles is partly lost. For more details regarding circulation time and biodistribution, readers can refer to other sources [46]. Regarding cellular interactions and uptake, liposomes can be equipped with targeting ligands, which can bind to receptors or other molecules that are specific or overexpressed by target cells for interactions and the intracellular delivery of drugs [46]. However, the drug delivery of liposomes is not efficient, since many modifications have been designed to minimize clearance and poisonousness. In general, liposomes accumulate in the macrophages of the liver and spleen after intravenous injection. Few liposomes are interspersed in other tissues, which may be due to the lack of immunocompatibility. On the other hand, exosomes are born with many features of an ideal drug delivery vehicle. For example, they exhibit lower toxicity compared to liposomes. In addition, they are well tolerated by the immune system, even across the blood-brain barrier, avoiding phagocytosis or degradation by macrophages [47]. Exosomes exhibit an innate targeting tendency. For instance, MSC-derived exosomes home preferentially to inflamed tissues and tumor tissues [48]. Furthermore, abundant bioactive materials within exosomes or on the surface provide primitive treatment potential, and there are abundant modification methods for membrane targeting and drug loading. Alvarez-Erviti et al. engineered dendritic cells to express Lamp2b, an exosomal membrane protein, fused to the neuron-specific RVG peptide and loaded these modified exosomes with siRNAs by electroporation. These intravenously injected exosomes showed a strong knockdown of BACE1 (mRNA (60\%) and protein $(62 \%))$, a therapeutic target of Alzheimer's disease, in wild-type mice [49].
4.2. Exosomal Modification and Cargo Loading. To amplify the therapeutic effects, many studies try to modify and load various treatment factors into exosomes via various methods. To date, these methods can be classified into two categories: (1) loading after isolation and (2) loading exosomes during biogenesis.

The first approach has been applied to load chemotherapeutic agents, siRNAs, and miRNAs. To reduce immunogenicity and toxicity of doxorubicin, Tian's group facilitated exosomal tumor targeting by engineering mouse immature dendritic cells (imDCs) to express a well-characterized exosomal membrane protein (Lamp2b) fused to a breast cancer-specific iRGD peptide (CRGDKGPDC). Chemotherapeutic agents were loaded via electroporation. The results showed an encapsulation efficiency of up to $20 \%$ and exosomal-delivered doxorubicin specific to breast cancer cells in vitro, leading to strong antiproliferative activity without overt toxicity after intravenous injection of BALB/C nude mice [50]. For nucleic acid, electroporation method has also been the first-rank used reported in several studies $[49,51]$. Although these studies provided positive delivery outcomes, debates remain. Some studies indicate that siRNA encapsulation is an illusion caused by nonspecific aggregate formation, independent of the exosomes. In addition, no significant encapsulation of siRNA could be measured when aggregate formation was blocked [52]. Therefore, it is necessary to establish multiple protocols for loading exosomes with nucleic acid.

The second approach is based on transfection methods to package active proteins, nucleic acid, and other active molecules into exosomes, where cells are transfected with an engineered effector-expressing vector. Liu's group used this method to load cells with opioid receptor Mu (MOR) siRNA in order to treat drug addiction via downregulating the expression of MOR, the primary target for opioid analgesics used clinically, including morphine, fentanyl, and methadone. This novel study provided a new strategy for the treatment of drug addiction [53]. Similarly, synthesized RNA oligonucleotides were transferred to MSCs in order to produce miR-143-rich exosomes, inhibiting the migratory potential of osteosarcoma cells [54]. Akt was transfected into umbilical cord-derived MSCs by using an adenovirus transfection system that improved cardiac function in animals treated with modified exosomes [55]. In addition, Pascucci reported that MSCs can acquire strong antitumor properties after incubation with paclitaxel (PTX), including the uptake of high drug doses followed by release into exosomes, inhibiting tumor growth activity. This method provides the possibility of using MSCs for the development of drugs with a higher cell-target specificity [56]. Sterzenbach reported the usage of the evolutionarily conserved latedomain (L-domain) pathway as a mechanism for loading exogenous proteins into exosomes [57]. They labeled an intracellular target protein with a WW tag, which was recognized by the L-domain motifs on Ndfip1, resulting in the loading of the target protein into exosomes.

For better tissue-targeting and an enhanced exosomal therapeutic effect, surface modification of exosomes was recently attempted by many groups using gene transfection 


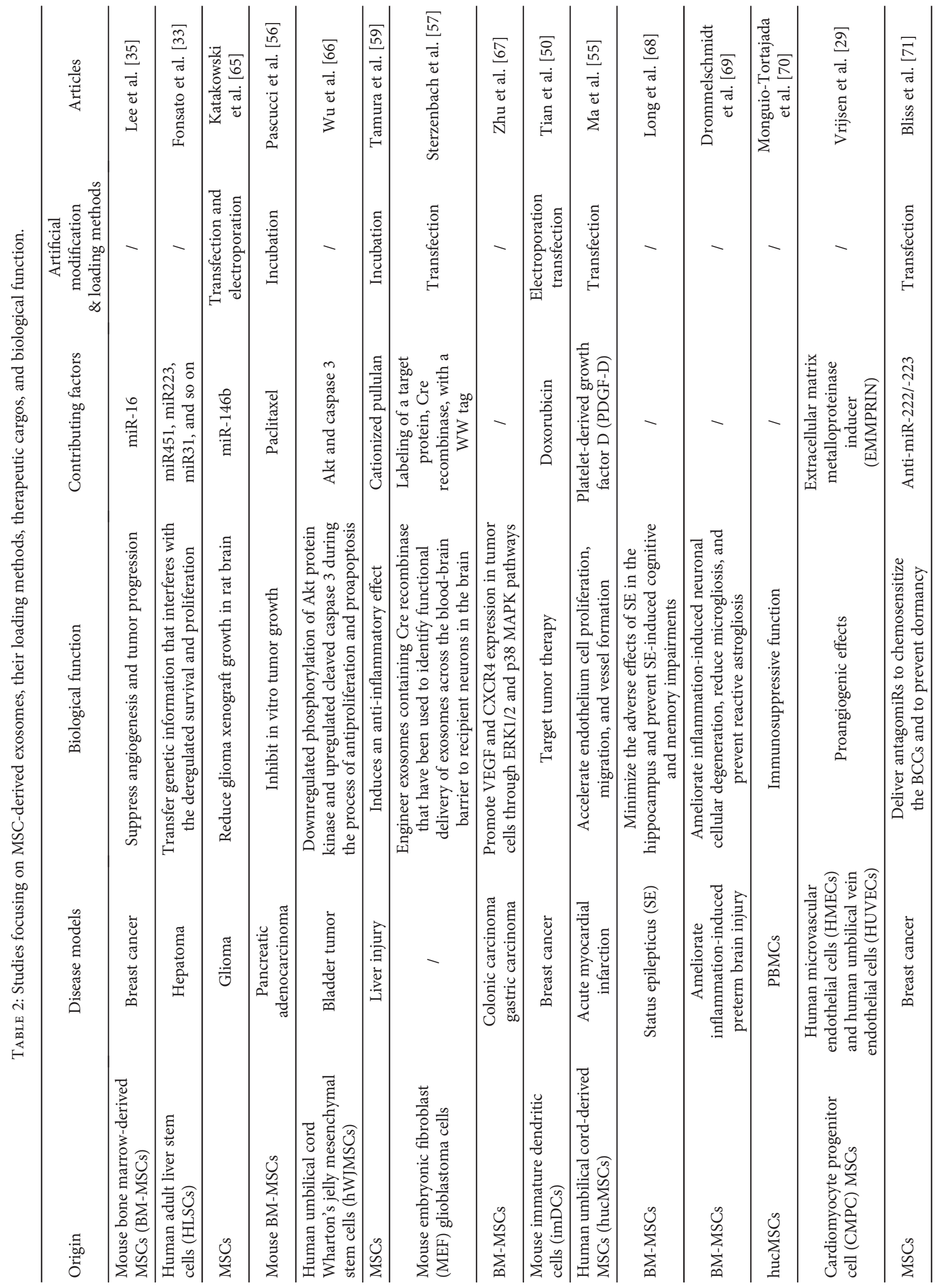




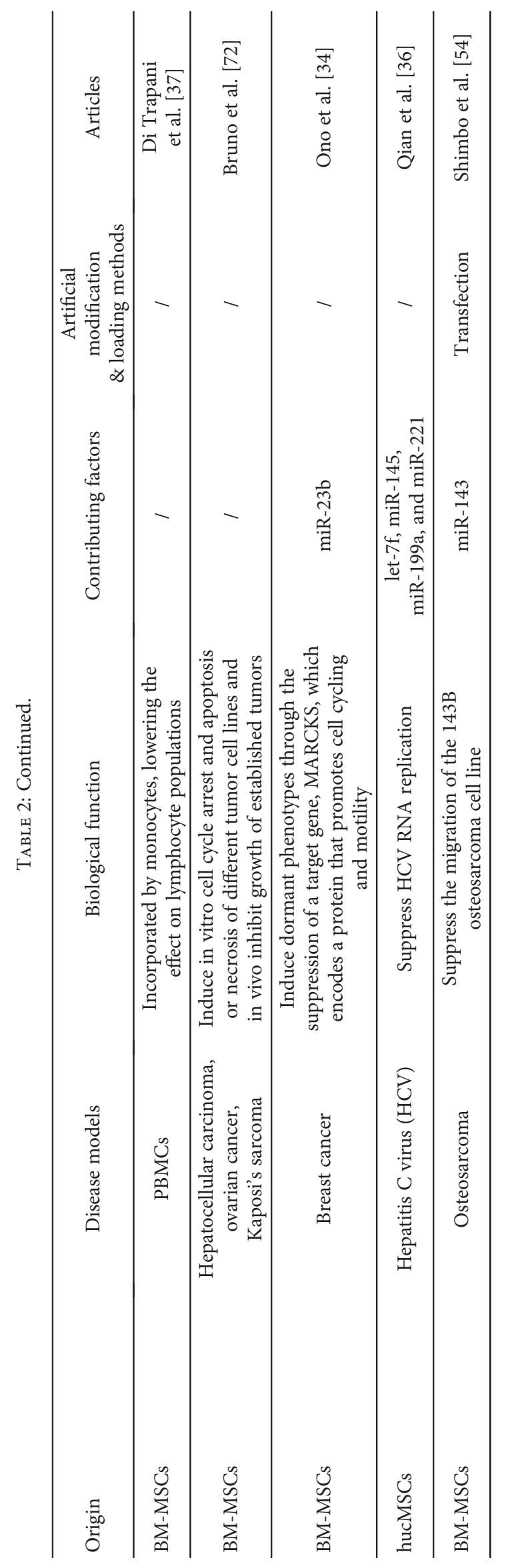


TABLE 3: Clinical trials of exosome-based therapies.

\begin{tabular}{|c|c|c|c|c|}
\hline Study title & Disease & Intervention & Phase & NCT \\
\hline $\begin{array}{l}\text { Effect of plasma-derived exosomes on } \\
\text { intractable cutaneous wound healing: } \\
\text { prospective trial }\end{array}$ & Ulcer & $\begin{array}{l}\text { Autologous exosomes } \\
\text { Rich plasma }\end{array}$ & Early phase 1 & NCT02565264 \\
\hline $\begin{array}{l}\text { Study investigating the ability of plant } \\
\text { exosomes to deliver curcumin to normal } \\
\text { and colon cancer tissue }\end{array}$ & Colon cancer & $\begin{array}{l}\text { Curcumin delivery } \\
\text { by exosomes }\end{array}$ & Phase 1 & NCT01294072 \\
\hline $\begin{array}{l}\text { Effect of microvesicles and exosomes therapy on } \\
\beta \text {-cell mass in type I diabetes mellitus (T1DM) }\end{array}$ & $\begin{array}{l}\text { Type I diabetes } \\
\text { mellitus }\end{array}$ & $\begin{array}{l}\text { Umbilical cord blood- } \\
\text { derived } \\
\text { MSC microvesicles }\end{array}$ & $\begin{array}{l}\text { Phase } 2 \\
\text { Phase } 3\end{array}$ & NCT02138331 \\
\hline $\begin{array}{l}\text { Preliminary clinical trial investigating the ability } \\
\text { of plant exosomes to abrogate oral mucositis } \\
\text { induced by combined chemotherapy and } \\
\text { radiation in head and neck cancer patients }\end{array}$ & $\begin{array}{l}\text { Head and neck cancer } \\
\text { Oral mucositis }\end{array}$ & $\begin{array}{l}\text { Dietary Supplement: } \\
\text { grape extract } \\
\text { Drug: lortab, fentanyl } \\
\text { patch, mouthwash }\end{array}$ & Phase 1 & NCT01668849 \\
\hline $\begin{array}{l}\text { Pilot study of metformin in head and neck } \\
\text { cancer and its effect on proinflammatory } \\
\text { cytokines and exosomes implicated in acute } \\
\text { and chronic toxicity }\end{array}$ & Head and neck cancer & $\begin{array}{l}\text { Radiation: external beam } \\
\text { radiation therapy } \\
\text { Drug: metformin } \\
\text { hydrochloride } \\
\text { Other: placebo }\end{array}$ & Early phase 1 & NCT03109873 \\
\hline
\end{tabular}

Phase II trial of a vaccination with tumor antigen-loaded dendritic cell-derived exosomes on patients with unresectable non-small-cell lung cancer responding to induction chemotherapy

Clinical trial of tumor cell-derived microparticles packaging chemotherapeutic drugs to treat malignant pleural effusion
Tumor antigen-loaded dendritic cell-derived exosomes

Malignant pleural effusion

Biological: tumor-derived

microparticles

Drug: cisplatin

Non-small-cell lung cancer
Phase 2 NCT01159288 


\section{Conclusion and Perspective}

The therapeutic potential of exosomes presents exciting new avenues for intervention in many diseases. The ability instinct to transport genetic messages and to protect the cargos to natural preferential recipient cells has drawn a rapid rise in attention. Therefore, specialized journals and websites have been established to disseminate this continuously unraveling information. While various clinical trials are underway to evaluate the safety and effectiveness of exosomes as therapeutic targets, many issues still remain. Questions regarding how clinical-grade exosomes can be produced in quantity and how various loading and isolation strategies impact the potency of exosome-based drug delivery remain unanswered. Therefore, there is an urgent need to closely examine the following aspects of exosomes: (1) natural therapeutic potential; (2) biogenesis mechanism; and (3) circulation kinetics and biodistribution. There is still a long road ahead, from promising phenomenological observations to clinical applications.

\section{Conflicts of Interest}

The authors declare that there is no conflict of interest regarding the publication of this article.

\section{Acknowledgments}

This study was supported by grants from the National Natural Science Foundation of China (81302242), Jilin Province Science and Technology Funds (20150204007YY and 20140204022YY), and Jilin Province Development and Reform Commission funds (2014G073 and 2016C046-2).

\section{References}

[1] C. Toma, W. R. Wagner, S. Bowry, A. Schwartz, and F. Villanueva, "Fate of culture-expanded mesenchymal stem cells in the microvasculature: in vivo observations of cell kinetics," Circulation Research, vol. 104, no. 3, pp. 398-402, 2009.

[2] A. M. Katsha, S. Ohkouchi, H. Xin et al., "Paracrine factors of multipotent stromal cells ameliorate lung injury in an elastaseinduced emphysema model," Molecular Therapy, vol. 19, no. 1, pp. 196-203, 2011.

[3] R. H. Lee, A. A. Pulin, M. J. Seo et al., "Intravenous hMSCs improve myocardial infarction in mice because cells embolized in lung are activated to secrete the anti-inflammatory protein TSG-6," Cell Stem Cell, vol. 5, no. 1, pp. 54-63, 2009.

[4] J. L. Spees, R. H. Lee, and C. A. Gregory, "Mechanisms of mesenchymal stem/stromal cell function," Stem Cell Research \& Therapy, vol. 7, no. 1, p. 125, 2016.

[5] M. Tkach and C. Thery, "Communication by extracellular vesicles: where we are and where we need to go," Cell, vol. 164, no. 6, pp. 1226-1232, 2016.

[6] S. R. Baglio, K. Rooijers, D. Koppers-Lalic et al., "Human bone marrow- and adipose-mesenchymal stem cells secrete exosomes enriched in distinctive miRNA and tRNA species," Stem Cell Research \& Therapy, vol. 6, no. 1, p. 127, 2015.

[7] S. Keerthikumar, D. Chisanga, D. Ariyaratne et al., "ExoCarta: a web-based compendium of exosomal cargo," Journal of Molecular Biology, vol. 428, no. 4, pp. 688-692, 2016.
[8] T. Skotland, K. Sandvig, and A. Llorente, "Lipids in exosomes: current knowledge and the way forward," Progress in Lipid Research, vol. 66, pp. 30-41, 2017.

[9] Y. Sato-Kuwabara, S. A. Melo, F. A. Soares, and G. A. Calin, "The fusion of two worlds: non-coding RNAs and extracellular vesicles - diagnostic and therapeutic implications (review)," International Journal of Oncology, vol. 46, no. 1, pp. 17-27, 2015.

[10] B. K. Thakur, H. Zhang, A. Becker et al., "Double-stranded DNA in exosomes: a novel biomarker in cancer detection," Cell Research, vol. 24, no. 6, pp. 766-769, 2014.

[11] M. Guescini, S. Genedani, V. Stocchi, and L. F. Agnati, “Astrocytes and glioblastoma cells release exosomes carrying mtDNA," Journal of Neural Transmission, vol. 117, no. 1, pp. 1-4, 2010.

[12] C. Kahlert, S. A. Melo, A. Protopopov et al., "Identification of double-stranded genomic DNA spanning all chromosomes with mutated KRAS and p53 DNA in the serum exosomes of patients with pancreatic cancer," The Journal of Biological Chemistry, vol. 289, no. 7, pp. 3869-3875, 2014.

[13] C. Harding, J. Heuser, and P. Stahl, "Receptor-mediated endocytosis of transferrin and recycling of the transferrin receptor in rat reticulocytes," The Journal of Cell Biology, vol. 97, no. 2, pp. 329-339, 1983.

[14] R. C. Lai, R. W. Y. Yeo, K. H. Tan, and S. K. Lim, "Exosomes for drug delivery-a novel application for the mesenchymal stem cell," Biotechnology Advances, vol. 31, no. 5, pp. 543551, 2013.

[15] C. Yang and P. D. Robbins, "The roles of tumor-derived exosomes in cancer pathogenesis," Clinical and Developmental Immunology, vol. 2011, Article ID 842849, 11 pages, 2011.

[16] C. Thery, S. Amigorena, G. Raposo, and A. Clayton, "Isolation and characterization of exosomes from cell culture supernatants and biological fluids," Current Protocols in Cell Biology, 2006, Chapter 3, Unit 322.

[17] D. Zabeo, A. Cvjetkovic, C. Lasser, M. Schorb, J. Lotvall, and J. L. Hoog, "Exosomes purified from a single cell type have diverse morphology," Journal of Extracellular Vesicles, vol. 6, no. 1, article 1329476, 2017.

[18] E. van der Pol, A. N. Boing, P. Harrison, A. Sturk, and R. Nieuwland, "Classification, functions, and clinical relevance of extracellular vesicles," Pharmacological Reviews, vol. 64, no. 3, pp. 676-705, 2012.

[19] G. Raposo, H. W. Nijman, W. Stoorvogel et al., "B lymphocytes secrete antigen-presenting vesicles," The Journal of Experimental Medicine, vol. 183, no. 3, pp. 1161-1172, 1996.

[20] P. Gutwein, A. Stoeck, S. Riedle et al., "Cleavage of L1 in exosomes and apoptotic membrane vesicles released from ovarian carcinoma cells," Clinical Cancer Research, vol. 11, no. 7, pp. 2492-2501, 2005.

[21] J. Paggetti, F. Haderk, M. Seiffert et al., "Exosomes released by chronic lymphocytic leukemia cells induce the transition of stromal cells into cancer-associated fibroblasts," Blood, vol. 126, no. 9, pp. 1106-1117, 2015.

[22] J. A. Cho, H. Park, E. H. Lim et al., "Exosomes from ovarian cancer cells induce adipose tissue-derived mesenchymal stem cells to acquire the physical and functional characteristics of tumor-supporting myofibroblasts," Gynecologic Oncology, vol. 123, no. 2, pp. 379-386, 2011. 
[23] M. F. Peterson, N. Otoc, J. K. Sethi, A. Gupta, and T. J. Antes, "Integrated systems for exosome investigation," Methods, vol. 87, pp. 31-45, 2015.

[24] S. Gholizadeh, M. Shehata Draz, M. Zarghooni et al., "Microfluidic approaches for isolation, detection, and characterization of extracellular vesicles: current status and future directions," Biosensors and Bioelectronics, vol. 91, pp. 588$605,2017$.

[25] C. Liu, J. Guo, F. Tian et al., "Field-free isolation of exosomes from extracellular vesicles by microfluidic viscoelastic flows," ACS Nano, vol. 11, no. 7, pp. 6968-6976, 2017.

[26] D. Kang, S. Oh, S. M. Ahn, B. H. Lee, and M. H. Moon, "Proteomic analysis of exosomes from human neural stem cells by flow field-flow fractionation and nanoflow liquid chromatography-tandem mass spectrometry," Journal of Proteome Research, vol. 7, no. 8, pp. 3475-3480, 2008.

[27] F. Caponnetto, I. Manini, M. Skrap et al., "Size-dependent cellular uptake of exosomes," Nanomedicine, vol. 13, no. 3, pp. 1011-1020, 2017.

[28] F. Arslan, R. C. Lai, M. B. Smeets et al., "Mesenchymal stem cell-derived exosomes increase ATP levels, decrease oxidative stress and activate PI3K/Akt pathway to enhance myocardial viability and prevent adverse remodeling after myocardial ischemia/reperfusion injury," Stem Cell Research, vol. 10, no. 3, pp. 301-312, 2013.

[29] K. R. Vrijsen, J. A. Maring, S. A. J. Chamuleau et al., "Exosomes from cardiomyocyte progenitor cells and mesenchymal stem cells stimulate angiogenesis via EMMPRIN," Advanced Healthcare Materials, vol. 5, no. 19, pp. 2555-2565, 2016.

[30] J. Burrello, S. Monticone, C. Gai, Y. Gomez, S. Kholia, and G. Camussi, "Stem cell-derived extracellular vesicles and immune-modulation," Frontiers in Cell and Developmental Biology, vol. 4, p. 83, 2016.

[31] S. Levy, S. C. Todd, and H. T. Maecker, "CD81 (TAPA-1): a molecule involved in signal transduction and cell adhesion in the immune system," Annual Review of Immunology, vol. 16, no. 1, pp. 89-109, 1998.

[32] S. Chandra, D. Vimal, D. Sharma, V. Rai, S. C. Gupta, and D. K. Chowdhuri, "Role of miRNAs in development and disease: lessons learnt from small organisms," Life Sciences, vol. 185, pp. 8-14, 2017.

[33] V. Fonsato, F. Collino, M. B. Herrera et al., "Human liver stem cell-derived microvesicles inhibit hepatoma growth in SCID mice by delivering antitumor microRNAs," Stem Cells, vol. 30, no. 9, pp. 1985-1998, 2012.

[34] M. Ono, N. Kosaka, N. Tominaga et al., "Exosomes from bone marrow mesenchymal stem cells contain a microRNA that promotes dormancy in metastatic breast cancer cells," Science Signaling, vol. 7, no. 332, article ra63, 2014.

[35] J. K. Lee, S. R. Park, B. K. Jung et al., "Exosomes derived from mesenchymal stem cells suppress angiogenesis by downregulating VEGF expression in breast cancer cells," PLoS One, vol. 8, no. 12, article e84256, 2013.

[36] X. Qian, C. Xu, S. Fang et al., "Exosomal microRNAs derived from umbilical mesenchymal stem cells inhibit hepatitis C virus infection," Stem Cells Translational Medicine, vol. 5, no. 9, pp. 1190-1203, 2016.

[37] M. Di Trapani, G. Bassi, M. Midolo et al., "Differential and transferable modulatory effects of mesenchymal stromal cellderived extracellular vesicles on T, B and NK cell functions," Scientific Reports, vol. 6, no. 1, article 24120, 2016.
[38] G. H. Cui, J. Wu, F. F. Mou et al., "Exosomes derived from hypoxia-preconditioned mesenchymal stromal cells ameliorate cognitive decline by rescuing synaptic dysfunction and regulating inflammatory responses in APP/PS1 mice," The FASEB Journal, 2017.

[39] Y. Zhang, M. S. Kim, B. Jia et al., "Hypothalamic stem cells control ageing speed partly through exosomal miRNAs," Nature, vol. 548, no. 7665, pp. 52-57, 2017.

[40] J. R. Chevillet, Q. Kang, I. K. Ruf et al., "Quantitative and stoichiometric analysis of the microRNA content of exosomes," Proceedings of the National Academy of Sciences of the United States of America, vol. 111, no. 41, pp. 14888-14893, 2014.

[41] P. Xia, S. Wang, B. Ye et al., "Sox2 functions as a sequencespecific DNA sensor in neutrophils to initiate innate immunity against microbial infection," Nature Immunology, vol. 16, no. 4, pp. 366-375, 2015.

[42] D. G. Phinney and M. F. Pittenger, "Concise review: MSCderived exosomes for cell-free therapy," Stem Cells, vol. 35, no. 4, pp. 851-858, 2017.

[43] C. Lo Sicco, D. Reverberi, C. Balbi et al., "Mesenchymal stem cell-derived extracellular vesicles as mediators of antiinflammatory effects: endorsement of macrophage polarization," Stem Cells Translational Medicine, vol. 6, no. 3, pp. 1018-1028, 2017.

[44] H. Xing, K. Hwang, and Y. Lu, "Recent developments of liposomes as nanocarriers for theranostic applications," Theranostics, vol. 6, no. 9, pp. 1336-1352, 2016.

[45] L. M. Mu, R. J. Ju, R. Liu et al., "Dual-functional drug liposomes in treatment of resistant cancers," Advanced Drug Delivery Reviews, vol. 115, pp. 46-56, 2017.

[46] R. van der Meel, M. H. Fens, P. Vader, W. W. van Solinge, O. Eniola-Adefeso, and R. M. Schiffelers, "Extracellular vesicles as drug delivery systems: lessons from the liposome field," Journal of Controlled Release, vol. 195, pp. 72-85, 2014.

[47] X. Zhuang, X. Xiang, W. Grizzle et al., "Treatment of brain inflammatory diseases by delivering exosome encapsulated anti-inflammatory drugs from the nasal region to the brain," Molecular Therapy, vol. 19, no. 10, pp. 1769-1779, 2011.

[48] B. Yu, X. Zhang, and X. Li, "Exosomes derived from mesenchymal stem cells," International Journal of Molecular Sciences, vol. 15, no. 3, pp. 4142-4157, 2014.

[49] L. Alvarez-Erviti, Y. Seow, H. Yin, C. Betts, S. Lakhal, and M. J. A. Wood, "Delivery of siRNA to the mouse brain by systemic injection of targeted exosomes," Nature Biotechnology, vol. 29, no. 4, pp. 341-345, 2011.

[50] Y. Tian, S. Li, J. Song et al., "A doxorubicin delivery platform using engineered natural membrane vesicle exosomes for targeted tumor therapy," Biomaterials, vol. 35 , no. 7, pp. 23832390,2014

[51] J. Wahlgren, T. D. L. Karlson, M. Brisslert et al., "Plasma exosomes can deliver exogenous short interfering RNA to monocytes and lymphocytes," Nucleic Acids Research, vol. 40, no. 17, article e130, 2012.

[52] P. Vader, S. A. A. Kooijmans, S. Stremersch, and K. Raemdonck, "New considerations in the preparation of nucleic acid-loaded extracellular vesicles," Therapeutic Delivery, vol. 5, no. 2, pp. 105-107, 2014.

[53] Y. Liu, D. Li, Z. Liu et al., "Targeted exosome-mediated delivery of opioid receptor $\mathrm{Mu}$ siRNA for the treatment of morphine relapse," Scientific Reports, vol. 5, no. 1, article 17543, 2015. 
[54] K. Shimbo, S. Miyaki, H. Ishitobi et al., "Exosome-formed synthetic microRNA-143 is transferred to osteosarcoma cells and inhibits their migration," Biochemical and Biophysical Research Communications, vol. 445, no. 2, pp. 381-387, 2014.

[55] J. Ma, Y. Zhao, L. Sun et al., "Exosomes derived from Akt-modified human umbilical cord mesenchymal stem cells improve cardiac regeneration and promote angiogenesis via activating platelet-derived growth factor D," Stem Cells Translational Medicine, vol. 6, no. 1, pp. 51-59, 2017.

[56] L. Pascucci, V. Cocce, A. Bonomi et al., "Paclitaxel is incorporated by mesenchymal stromal cells and released in exosomes that inhibit in vitro tumor growth: a new approach for drug delivery," Journal of Controlled Release, vol. 192, pp. 262270, 2014.

[57] U. Sterzenbach, U. Putz, L. H. Low, J. Silke, S. S. Tan, and J. Howitt, "Engineered exosomes as vehicles for biologically active proteins," Molecular Therapy, vol. 25, no. 6, pp. 12691278, 2017.

[58] S. Ohno, M. Takanashi, K. Sudo et al., "Systemically injected exosomes targeted to EGFR deliver antitumor microRNA to breast cancer cells," Molecular Therapy, vol. 21, no. 1, pp. 185-191, 2013.

[59] R. Tamura, S. Uemoto, and Y. Tabata, “Augmented liver targeting of exosomes by surface modification with cationized pullulan," Acta Biomaterialia, vol. 57, pp. 274-284, 2017.

[60] M. Kanada, M. H. Bachmann, J. W. Hardy et al., "Differential fates of biomolecules delivered to target cells via extracellular vesicles," Proceedings of the National Academy of Sciences of the United States of America, vol. 112, pp. E1433-E1442, 2015.

[61] T. Chen, F. Arslan, Y. Yin et al., "Enabling a robust scalable manufacturing process for therapeutic exosomes through oncogenic immortalization of human ESC-derived MSCs," Journal of Translational Medicine, vol. 9, no. 1, p. 47, 2011.

[62] R. W. Y. Yeo, R. C. Lai, B. Zhang et al., "Mesenchymal stem cell: an efficient mass producer of exosomes for drug delivery," Advanced Drug Delivery Reviews, vol. 65, no. 3, pp. 336-341, 2013.

[63] L. Sun, R. Xu, X. Sun et al., "Safety evaluation of exosomes derived from human umbilical cord mesenchymal stromal cell," Cytotherapy, vol. 18, no. 3, pp. 413-422, 2016.

[64] R. Munagala, F. Aqil, J. Jeyabalan, and R. C. Gupta, "Bovine milk-derived exosomes for drug delivery," Cancer Letters, vol. 371, no. 1, pp. 48-61, 2016.

[65] M. Katakowski, B. Buller, X. Zheng et al., "Exosomes from marrow stromal cells expressing miR-146b inhibit glioma growth," Cancer Letters, vol. 335, no. 1, pp. 201-204, 2013.

[66] S. Wu, G. Q. Ju, T. Du, Y. J. Zhu, and G. H. Liu, "Microvesicles derived from human umbilical cord Wharton's jelly mesenchymal stem cells attenuate bladder tumor cell growth in vitro and in vivo," PLoS One, vol. 8, no. 4, article e61366, 2013.

[67] W. Zhu, L. Huang, Y. Li et al., "Exosomes derived from human bone marrow mesenchymal stem cells promote tumor growth in vivo," Cancer Letters, vol. 315, no. 1, pp. 28-37, 2012.

[68] Q. Long, D. Upadhya, B. Hattiangady et al., "Intranasal MSC-derived A1-exosomes ease inflammation, and prevent abnormal neurogenesis and memory dysfunction after status epilepticus," Proceedings of the National Academy of Sciences of the United States of America, vol. 114, no. 17, pp. E3536E3545, 2017.

[69] K. Drommelschmidt, M. Serdar, I. Bendix et al., "Mesenchymal stem cell-derived extracellular vesicles ameliorate inflammation-induced preterm brain injury," Brain, Behavior, and Immunity, vol. 60, pp. 220-232, 2017.

[70] M. Monguio-Tortajada, S. Roura, C. Galvez-Monton et al., "Nanosized UCMSC-derived extracellular vesicles but not conditioned medium exclusively inhibit the inflammatory response of stimulated $\mathrm{T}$ cells: implications for nanomedicine," Theranostics, vol. 7, no. 2, pp. 270-284, 2017.

[71] S. A. Bliss, G. Sinha, O. A. Sandiford et al., "Mesenchymal stem cell-derived exosomes stimulate cycling quiescence and early breast cancer dormancy in bone marrow," Cancer Research, vol. 76, no. 19, pp. 5832-5844, 2016.

[72] S. Bruno, F. Collino, M. C. Deregibus, C. Grange, C. Tetta, and G. Camussi, "Microvesicles derived from human bone marrow mesenchymal stem cells inhibit tumor growth," Stem Cells and Development, vol. 22, no. 5, pp. 758-771, 2013. 

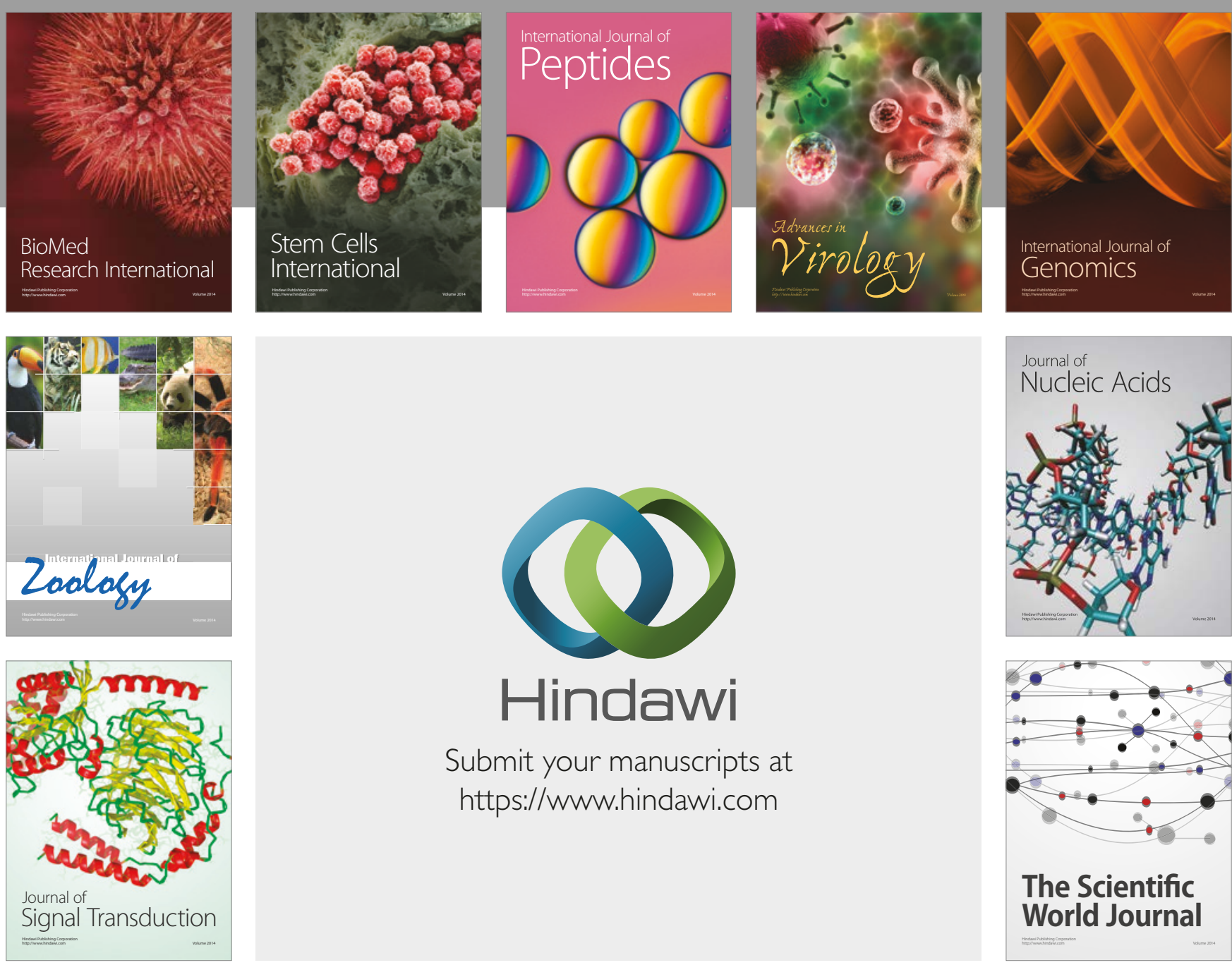

Submit your manuscripts at

https://www.hindawi.com
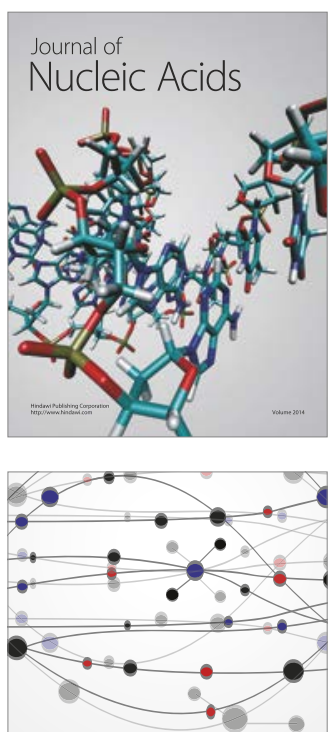

The Scientific World Journal

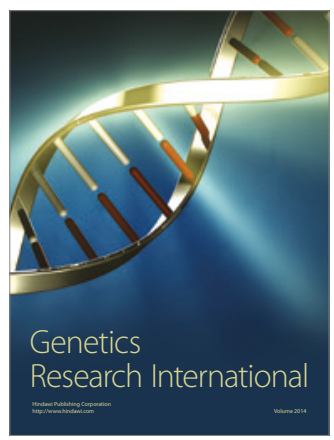

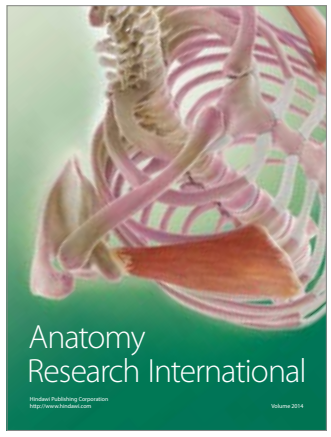

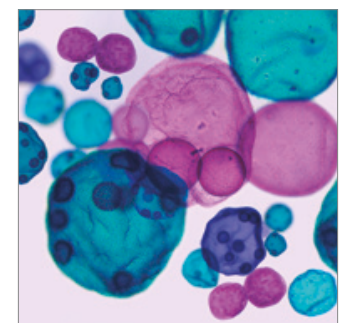

International Journal of Microbiology
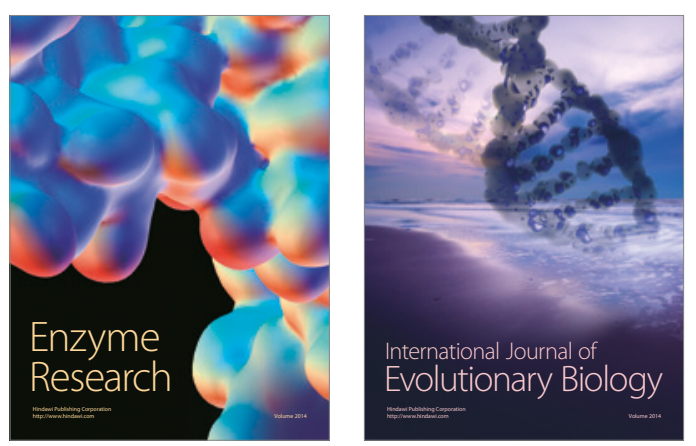
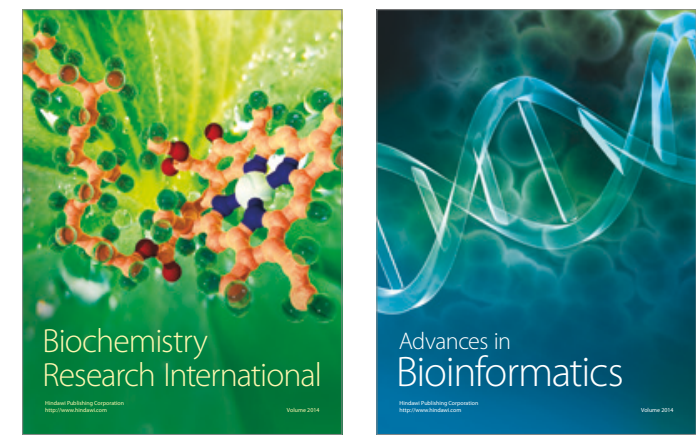

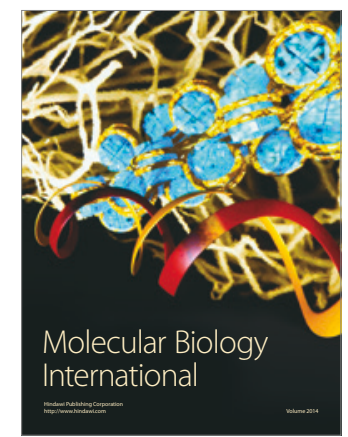

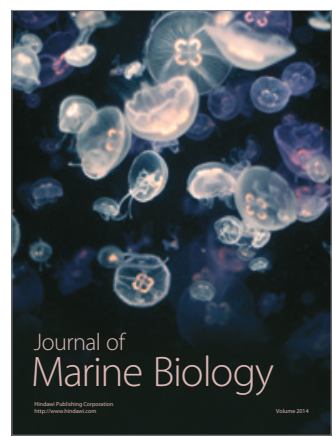

